\title{
Nanohardness and Microstructure of NiCoAlFeCu and NiCoAlFeCuCr Alloys Produced by Mechanical Alloying
}

C. D. Gómez-Esparza ${ }^{1}$, K. Campos-Venegas ${ }^{1}$, O. Solis-Canto ${ }^{1}$, J.M. Alvarado-Orozco ${ }^{2}$, J. MuñozSaldaña $^{2}$, J. M. Herrera-Ramírez ${ }^{1}$ and R. Martínez-Sánchez ${ }^{1}$

${ }^{1}$ Centro de Investigación en Materiales Avanzados (CIMAV), Laboratorio Nacional de Nanotecnología, Miguel de Cervantes 120, 31109 Chihuahua, Chih., México.

${ }^{2}$ Centro de Investigación y de Estudios Avanzados del IPN, Unidad Querétaro, Querétaro, 76230, México.

The development of high-entropy (HE) alloys expanded the path of advanced materials research. These alloys with at least 5 principal elements in equiatomic or near equiatomic ratios have the tendency to form solid solutions (SS) phases, usually with FCC and/or BCC structures, instead of intermetallic compounds [1]. This effect is reflected with superior mechanical properties compared with conventional alloys which are composed for one or two principal elements [2]. The structures and microstructures in HE alloys, as well as their mechanical properties depend on the nature and number of the alloying elements. The mechanical properties of several high-entropy alloys have been reported. However, evaluations by nanoindentation in $\mathrm{HE}$ alloys have not been deeply investigated. The $\mathrm{AlCoCrCuFeNi}$ alloy has been the most widely investigated in terms of microstructure and mechanical properties, but at this moment there are no reports about hardness determinations of individual phases. This paper first reviews the microstructure of high entropy alloys in relation to their chemical composition, particularly to the contribution of $\mathrm{Cr}$ adittion, and its effect on microhardness of bulk samples and the mechanical properties of individual phases by nanoindentation tests.

Equiatomic $\mathrm{NiCoAlFeCu}$ and $\mathrm{NiCoAlFeCuCr}$ high entropy alloys were prepared by mechanical alloying during $10 \mathrm{~h}$ under an argon atmosphere in a SPEX $8000 \mathrm{M}$ ball mill. High-purity elemental powders were used as starting materials. The as-milled powders were cold compacted in a uniaxial press with a load of $1500 \mathrm{MPa}$, followed by conventional sintering process at $1200{ }^{\circ} \mathrm{C}$ during $3 \mathrm{~h}$ in vacuum. The microhardness tests were performed in a Vickers CLEMEX MMTX-7 tester, with a normal load of $200 \mathrm{~g}$ and a dwell time of $10 \mathrm{~s}$. Nanoindentation studies were performed on bulk samples with a Berkovich (three-sided pyramidal) diamond tip using a nanomechanical test instrument (Ubil, Hysitron, USA) equipped with in-situ Scanning Probe Microscopy (SPM) imaging that provides high-resolution SPM images of the test location, which is useful to study multiphase samples. The probe can be place in the desired position of the sample.

According to XRD results the two alloys exhibit the presence of two-phase mixture FCC + BCC (Fig. 1). The EDS/SEM and mapping analyses show the presence of a $\mathrm{Cu}$-rich SS phase and a SS phase with high $\mathrm{Ni}$, Co and $\mathrm{Al}$ content in the quinary alloy (Fig. 2). Besides the two mentioned phases, the addition of $\mathrm{Cr}$ promotes the formation of a third Cr-rich phase (Fig. 3). The microhardness measurements present a wide standard deviation due to the typical porosity density of mechanically alloyed samples. A slightly increase in microhardness occurred with the $\mathrm{Cr}$ addition. On the other hand, the mechanical properties of individual phases demonstrated an evident dependence with chemical distribution, structure and microstructure. The NiCoAlFeCuCr alloy exhibits the highest nanohardness values, the maximum hardness $(\sim 20 \mathrm{GPa})$ was achieved with the Cr-rich solid solution phase. Nevertheless, this phase is 
present in a lower volume fraction than the other two phases, this effect is reflected in similar general hardness to the NiCoAlFeCu alloy.

\section{References:}

[1] J.-W. Yeh, S.-J. Lin, T.-S. Chin, J.-Y. Gan, S.-K. Chen, T.-T. Shun, C.-H. Tsau, S.-Y. Chou, Metall. Mater. Trans. A, 35 (2004) p. 2533-2536.

[2] S.-T. Chen, W.-Y. Tang, Y.-F. Kuo, S.-Y. Chen, C.-H. Tsau, T.-T. Shun, J.-W. Yeh, Mater. Sci. Eng. A, 527 (2010) p. 5818-5825.

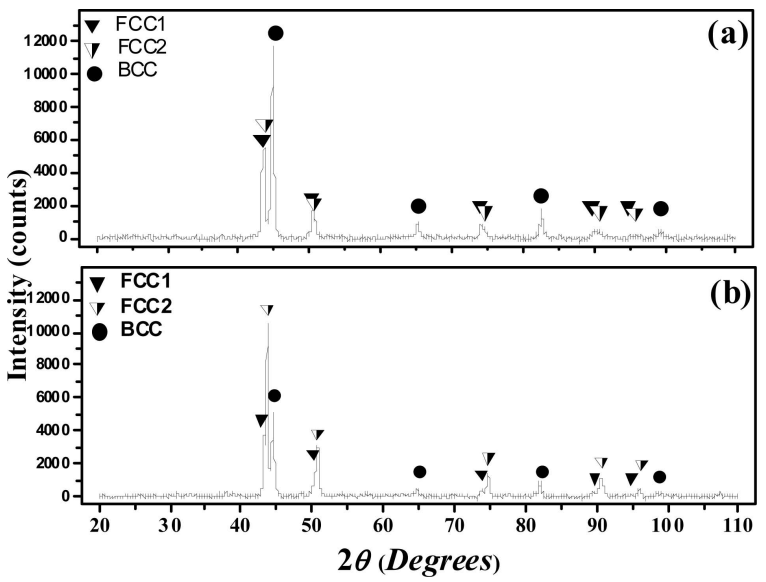

Figure 1. XRD patterns of: a) $\mathrm{NiCoAlFeCu}$ and b) $\mathrm{NiCoAlFeCuCr}$ alloys.

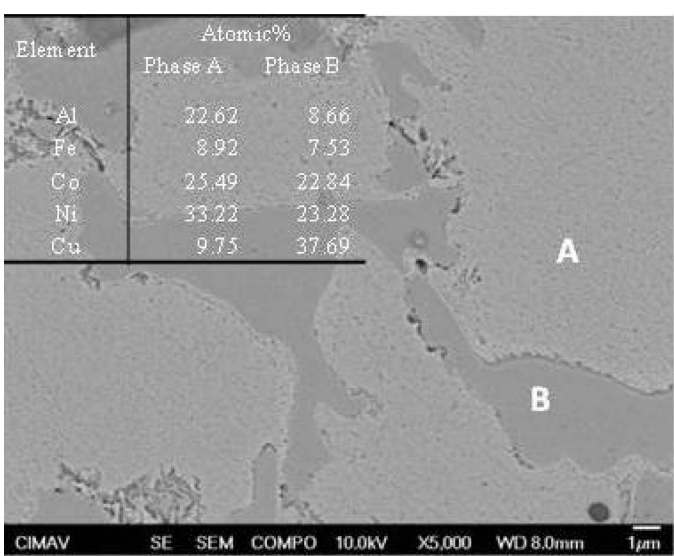

Figure 2. BE-SEM image of the $\mathrm{NiCoAlFeCu}$ alloy and the chemical composition of found phases by EDS-SEM.
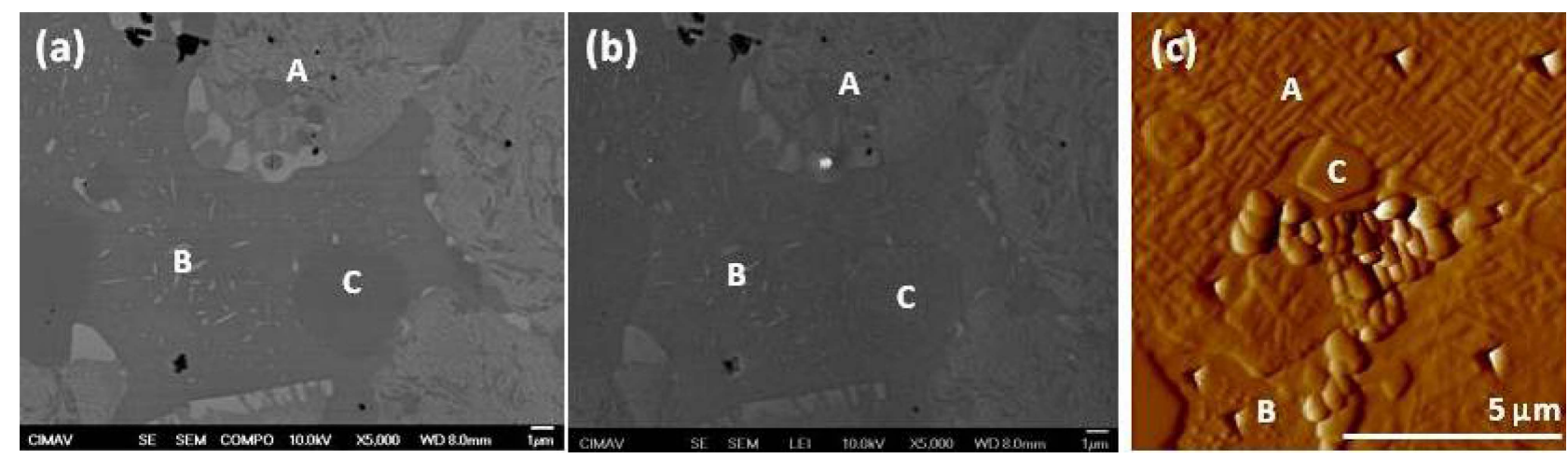

Figure 3. Micrographs of the NiCoAlFeCuCr alloy: (a) BE-SEM, (b) SE-SEM. (c) SPM nanoindentation impression images. Phase indicated as $\mathrm{C}$ corresponds to the $\mathrm{Cr}$-rich phase.

Table 1. Microhardness and mechanical properties of individual phases of: 1) NiCoAlFeCu and 2) NiCoAlFeCuCr bulk samples.

\begin{tabular}{lllllll}
\hline \multirow{2}{*}{ Alloy Phase } & & Structure & $\begin{array}{l}\text { Microha } \\
\text { rness } \\
(\mathbf{H V})\end{array}$ & $\begin{array}{l}\text { Nanohardness } \\
(\mathbf{H V}) \\
\text { GPA }\end{array}$ & $\begin{array}{l}\text { Er } \\
\text { (GPa) }\end{array}$ \\
\hline \multirow{2}{*}{1} & A & SS FCC (Ni-like) + Precip. FCC & $261 \pm 31$ & 501 & 4.92 & 158 \\
& B & SS BCC (Fe-like) & 751 & 7.37 & 175 \\
\hline \multirow{2}{*}{2} & A & SS FCC (Ni-like) + Precip. FCC & & 590 & 5.79 & 187 \\
& B & SS BCC (Fe-like) & $269 \pm 39$ & 833 & 8.17 & 173 \\
& C & Cr-rich SS & & 2102 & 20.61 & 265 \\
\hline
\end{tabular}

